\title{
Analytic continuation as a bridge between continuum and bound states
}

\author{
Leonid Blokhintsev ${ }^{1, a}$ and Dmitry Savin ${ }^{1, b}$ \\ ${ }^{1}$ Skobeltsyn Institute of Nuclear Physics, Lomonosov Moscow State University, Moscow, Russia
}

\begin{abstract}
The problem of obtaining characteristics of bound nuclear states from continuum states data is discussed. It is shown that the ambiguities due to the existence of phase-equivalent potentials can be resolved by using the analytic properties of scattering amplitudes. The methods of determination of asymptotic normalization coefficients and vertex constants are considered. The asymptotic normalization coefficients for ${ }^{6} \mathrm{Li}$ in the $\alpha+d$ channel are found by analytic continuation of the two-channel effective range expansion. The account of inelastic channels within the effective range approach is discussed.
\end{abstract}

\section{Introduction}

Asymptotic normalization coefficients (ANC) determine the asymptotics of nuclear wave functions in binary channels. ANCs are proportional to vertex constants (VC), which determine the virtual processes $A \rightarrow B+C[1]$.

VCs and ANCs are fundamental nuclear characteristics. They are used actively in analyses of nuclear reactions within various approaches. VCs and ANCs extracted from one process can be used for the prediction of characteristics of other processes. Comparing of empirical values of VCs and ANCs with theoretical ones enables one to evaluate the quality of a model. The ANC for the channel $A \rightarrow B+C$ determines the probability of the configuration $B+C$ in the nucleus $A$ at distances greater than the radius of nuclear interaction.

Thus ANCs arise naturally in the expressions for cross sections of nuclear reactions between charged particles at low energies, in particular, of astrophysical nuclear reactions.

Note that due to the Coulomb barrier the cross sections $\sigma(E)$ at astrophysical energies are so small that their direct measurement in laboratories is very difficult, or even impossible. At low energies

$$
\sigma(E) \propto \frac{1}{k} \frac{2 \pi \eta}{e^{2 \pi \eta}-1}
$$

where $\eta=Z_{B} Z_{C} e^{2} \mu / k$ is the Coulomb parameter for the initial $B+C$ state, $Z_{i} e$ is the charge of the particle $i, \mu$ is the reduced mass, and $k$ is the relative momentum.

Usually at astrophysical energies one uses the astrophysical $S$ factor in place of the cross section

$$
S(E)=E e^{2 \pi \eta} \sigma(E), \quad E=k^{2} / 2 \mu
$$

\footnotetext{
ae-mail: blokh@srd.sinp.msu.ru

be-mail: rhdp@mail.ru
} 
It was shown [2] that the cross section of the $B(C, \gamma) A$ reaction at astrophysical energies with a good accuracy is determined by the value of the ANC $C_{A B C}$ in the $B+C$ channel. This conclusion made it possible to calculate $S(E=0)$ for a number of radiative capture processes: ${ }^{4} \mathrm{He}(\mathrm{d}, \gamma)^{6} \mathrm{Li}$, ${ }^{4} \mathrm{He}\left({ }^{3} \mathrm{He}, \gamma\right){ }^{7} \mathrm{Be},{ }^{7,9} \mathrm{Be}(\mathrm{p}, \gamma){ }^{8,10} \mathrm{~B},{ }^{8} \mathrm{~B}(\mathrm{p}, \gamma){ }^{9} \mathrm{C},{ }^{11,13} \mathrm{C}(\mathrm{p}, \gamma){ }^{12,14} \mathrm{~N},{ }^{12-14} \mathrm{~N}(\mathrm{p}, \gamma){ }^{13-15} \mathrm{O},{ }^{17} \mathrm{~F}(\mathrm{p}, \gamma){ }^{18} \mathrm{Ne}$, ${ }^{20} \mathrm{Ne}(\mathrm{p}, \gamma){ }^{21} \mathrm{Na}$.

\section{Definition and Properties of ANCs and VCs}

For the short-range interaction the asymptotics of the radial overlap integral $I_{A B C}(L S ; r)$ of the wave functions of $A, B$, and $C$ is of the form

$$
\left.I_{A B C}(L S ; r)\right|_{r \rightarrow \infty}=C_{A B C}(L S) \frac{e^{-\varkappa r}}{r},
$$

where $\varkappa^{2}=2 \mu \epsilon, \epsilon=m_{B}+m_{C}-m_{A}, L(S)$ is the channel orbital momentum (channel spin). If the Coulomb interaction is present, then one should substitute the Whittaker function $W_{-\eta, L+1 / 2}(2 \varkappa r)$ for $e^{-x r}$.

$\operatorname{VC~} G_{A B C}(L S)$ is the on-shell matrix element of the virtual $A \leftrightarrow B+C$ process in the given partialwave state $L S$. It is related to the amplitude of elastic $B C$ scattering:

$$
\text { res }<L S\left|M^{J_{A}}\right| L S>\left.\right|_{E=-\epsilon}=(-1)^{L} G_{A B C}^{2}(L S)
$$

$G_{A B C}$ and $C_{A B C}$ are interrelated:

$$
G_{A B C}(L S)=-\left(\pi N_{B C}\right)^{1 / 2} C_{A B C}(L S) / \mu
$$

The factor $N_{B C}$ arises due to the identity of nucleons and depends on the way of the antisymmetrization of wave functions.

$$
1 \leq N_{B C} \leq \frac{\left(A_{B}+A_{C}\right) !}{A_{B} ! A_{C} !}
$$

Often $N_{B C}$ is included into $C_{A B C}$.

Note that the asymptotics (3) can be rigorously proved for two-body systems only. For threeand more particle systems the asymptotics of overlap integrals may differ from (3) ('anomalous' asymptotics) $[3,4]$.

Considering the Fourier component of the overlap integral $I(r)$ one gets

$$
\left.I(r)\right|_{r \rightarrow \infty}=C_{0} \frac{e^{-\varkappa r}}{r}+C_{1} \frac{e^{-\varkappa_{1} r}}{r^{p}}, \quad p>1
$$

where $i \varkappa_{1}$ is the nearest to the origin singular point of the vertex function $G(q)$ for the $A \rightarrow B+C$ vertex. If $\varkappa_{1}<\varkappa$, then the second term in (7) dominates at $r \rightarrow \infty$. Consider the diagram of figure 1, which contributes to $G(q)$.

This diagram results from the Faddeev expansion in the simplest three-body model, in which $A$ consists of $d, f$ and $C, B$ is a bound state of $d$ and $f$, and $e$ is a bound state of $f$ and $C$.

For that diagram $p=2$ and the singular point is

$$
q=i \varkappa_{1}=i \frac{m_{B}}{m_{d}}\left(\varkappa_{A d e}+\varkappa_{B d f}\right), \quad \varkappa_{i j k}=2 \mu_{j k} \epsilon_{i j k}, \quad \epsilon_{i j k}=m_{j}+m_{k}-m_{i}
$$

At any $\epsilon_{A B C}$ the 'anomalous' condition $\varkappa_{1}<\varkappa$ could be satisfied if $\epsilon_{A d e}$ and $\epsilon_{B d f}$ are sufficiently small. 


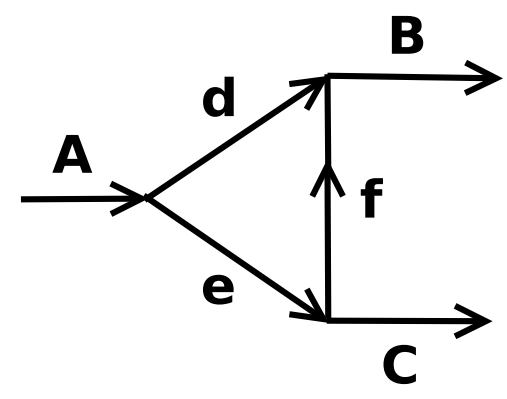

Figure 1.

\section{Methods of determining ANCs and VCs}

Microscopic calculations are very tedious.

Using the coordinate representation one should go to the asymptotical region where the values of wave functions are very small what results in low accuracy.

On the other hand, in the momentum representation to calculate VCs and ANCs one deals with imaginary values of momenta what complicates calculations considerably.

To our knowledge, there are only two works, in which ANCs for nuclear systems with $A>3$ were calculated ab initio:

- in the work [5] the ANCs for an alpha-particle were calculated using the hyperspherical harmonics approach.

- in the work [6] the ANCs for one-nucleon removals from nuclei with $3 \leq A \leq 9$ were obtained using the GFMC method.

The alternative way of getting ANCs and VCs is the analysis of experimental data on scattering and reaction processes. The examples are as follows

- If the pole mechanism (figure 2) contributes to the $A(x, y) B$ reaction, then the differential cross section $\sigma(z)$ of that reaction possesses the 2 nd order pole at $z=z_{0}\left(z=\cos \theta,\left|z_{0}\right|>1\right)$. If one extrapolates the experimental values of $\left(z-z_{0}\right)^{2} \sigma(z)$ to the pole position, one immediately obtains the value of $\left|G_{A B C} G_{y x C}\right|^{2}$.

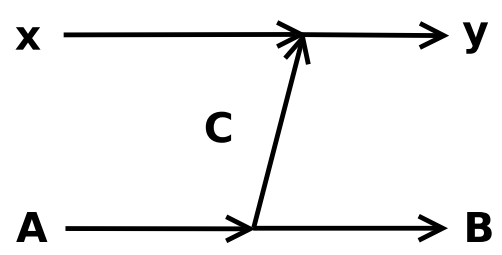

Figure 2.

- Extrapolation in energy $E$ of the partial-wave amplitude of elastic $B C$ scattering (obtained by the phase-shift analysis) to the pole corresponding to the bound state $A$ (figure 3 ) gives the value of $\left|G_{A B C}\right|^{2}$.

The problem of using continuum-state data to obtain information on bound-state characteristics is not trivial. 


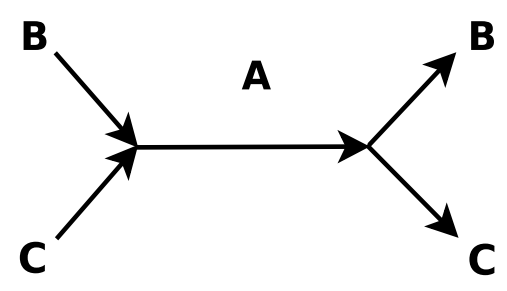

Figure 3.

"Bound-state properties cannot be extracted from the phase shifts of a single partial wave, as a matter of principle" ([7]; repeated in some original papers).

This assertion is based on the existence of phase-equivalent potentials (PEPs). Different PEPs lead to coinciding phase shifts $\delta_{L}(E)$ but properties of the bound states for a given $L$ are different.

According to the inverse scattering problem, to restore a local potential one needs to know

i) $\delta_{L}(E), 0 \leq E<\infty$.

ii) two additional parameters for each of $N_{L}$ bound states for a given $L$. One can choose $N_{L}$ binding energies and $N_{L}$ ANCs as such parameters.

Hence. within the formal potential approach with arbitrary potentials and without any additional conditions, it is impossible to determine unambiguously characteristics of bound states knowing only $\delta_{L}(E)$.

The way to resolve that ambiguity problem is to make use of the natural requirement that amplitudes of processes are analytic functions of their kinematic variables. As is known, the analyticity of amplitudes follows from the microcausality principle.

Using analiticity and knowing the partial-wave $B C$ scattering amplitude $f_{L}(E)$ on some segment of the real positive semiaxis, one can continue analytically $f_{L}(E)$ to the unphysical region $E<0$ and obtain both the position of the pole $E=-\epsilon<0$ and the residue of $f_{L}(E)$ at that pole, that is, the VC and ANC.

Note that in the present section we discuss the principal side of the problem and not the practical ways of analytic continuation.

Knowing $\epsilon$, ANC $C_{A B C}$, and $f_{L}(E)$ at $0 \leq E<\infty$, one can construct unambiguously the local potential $V(r)$ using methods of the inverse scattering problem. As a result, the unique "analytic" potential would be selected out of set of PEPs, which leads to the needed analytic properties of the scattering amplitude. That potential describes all bound and continuum states of a given system.

The existence of PEPs means that the characteristics of a bound state obtained by the direct analytic continuation of $f_{L}(E)$ from $E \geq 0$ to $E<0$ may differ from the characteristics found by solving the bound state problem with the potential which describes correctly $f_{L}(E)$ at $E \geq 0$. The cause of this fact can be elucidated as follows.

In the potential scattering theory the partial-wave amplitude is written as

$$
f_{L}(E)=-\frac{\mu}{2 \pi} \int_{0}^{\infty} d r \phi_{L}(k r) V(r) \psi_{L}(k r),
$$

where $\psi_{L}$ is the exact solution of the radial Schrödinger equation and $\phi_{L}$ is a plane wave. If $k$ is continued to the complex plane, the terms $e^{2|\operatorname{Im} \varkappa| r} V(r)$ arise in the integrand, which leads to the divergence of the integral if $V(r)$ does not decrease rapidly enough at $r \rightarrow \infty$. 
$f_{L}(k)$ in the form (9) can be analytically continued to $k=i \varkappa(E=-\epsilon)$ if ([7])

$$
\int_{0}^{\infty} d r r|V(r)| e^{2|x| r}<\infty
$$

If the condition (10) is violated, then the characteristics of bound states corresponding to the potential $V(r)$ cannot be obtained by using Eq.(9).

However, there exist other possibilities of analytic continuation of amplitudes, for instance:

- if the explicit form of $f_{L}(E)$ at $E \geq 0$ is known

- if one succeeds in approximating $f_{L}(E)$ at $E \geq 0$ by a certain analytic expression accurately enough.

Consider the trivial example:

$$
\chi(z)=\int_{0}^{\infty} e^{(a-z) t} d t
$$

Initially $\chi(z)$ is defined only at $\operatorname{Re} z>\operatorname{Re} a$ since if this inequality is violated, the integral diverges.

On the other hand, the integration in (11) can be performed explicitly giving

$$
\chi(z)=\frac{1}{z-a}
$$

This expression defines the function, which is analytic in the whole complex $z$ plane with a pole at $z=a$.

The description of elastic scattering of composite objects (say, nuclei) could be considered formally in the two-body potential approach, however, the corresponding potentials are complex, nonlocal and energy- and angular momentum dependent. Nevertheless, one can still use analytic continuation of $f_{L}(E)$ to $E<0$ to find the binding energy and the VC and ANC. Analytic continuation can be performed in different ways.

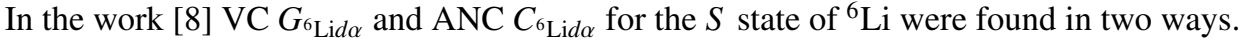

1. Analytic approximation of experimental values of $k \cot \delta$ using Padé approximants and subsequent continuation to $E<0$.

2. Constructing the effective two-body $d \alpha$ potential $V_{d \alpha}(r)$ describing experimental phase shifts and finding the two-body bound-state wave function for this potential. $V_{d \alpha}(r)$ was written as a sum of gaussians and satisfied the necessary condition (10).

The results of two different methods are in close agreement.

Note that in the general case, when $B$ and/or $C$ are composite systems, the ANC $C_{A B C}$ corresponds to the overlap integral $I_{A B C}(r)$, which is normalized not to 1 but to the spectroscopic factor $S_{A B C}$. However, if the ANC is found in the two-body model, the corresponding two-body bound-state wave function should be normalized to 1 . Normalizing this function to the independently determined spectroscopic factor is incorrect.

One may conclude that using the fundamental analyticity property of scattering amplitudes and analytic continuation methods allows one to obtain information on characteristics of bound states (including ANCs) from the phase shift data. Thus the ambiguity related to the existence of phaseequivalent potentials is removed.

\section{Analytic continuation of effective range expansion (ERE)}

One of the most widespread methods is the analytic continuation in energy of the data on the partialwave amplitude of elastic $B C$ scattering to the pole corresponding to the bound state $A$. The most 
effective way of realization of this procedure is the analytic continuation of the effective range function $K_{L}\left(k^{2}\right)$. This method was used in [8] to obtain the $S$ wave VCs and ANCs for the process ${ }^{6} \mathrm{Li} \rightarrow \alpha+d$, by Yu.V. Orlov et al. [9] for the systems ${ }^{3} \mathrm{H},{ }^{2,3,5} \mathrm{He},{ }^{5} \mathrm{Li},{ }^{8} \mathrm{Be}$, and by J.-M. Sparenberg et al. [10] for the systems ${ }^{16} \mathrm{O}+n,{ }^{16} \mathrm{O}+p$, and ${ }^{12} \mathrm{C}+\alpha$.

All above-listed works treated one-channel elastic scattering. However, the description of scattering of particles with nonzero spins even in the absence of inelastic channels often demands account of channel coupling. The most typical situation induced by tensor forces is the case of two coupled channels 1 and 2 with the same $J$ but different $L\left(L_{1}\right.$ and $\left.L_{2}=L_{1}+2\right)$. The examples are $d \alpha$ scattering, $N N$ triplet scattering etc. In principle, coupled channels may differ not in $L$ but in channel spins.

In the work [11] it was considered the generalization of the ERE to the case of two coupled channels and using that expansion for determining VCs and ANCs. The consideration in [11] was carried out for the short-range interaction, which practically limited using the formalism developed to the reactions induced by neutrons. Then the results of [11] were generalized to account of the Coulomb interaction which radically changes analytic properties of scattering amplitudes and their behavior at low energies.

The formalism developed could be applied to any two-channel nuclear system, for which the results of the phase-shift analysis are known (including the mixing parameter). One of similar important systems is ${ }^{6} \mathrm{Li}$ in the $\alpha+d$ channel. The ANC values for this system determine the cross section of the radiative capture ${ }^{4} \mathrm{He}(d, \gamma)^{6} \mathrm{Li}$, which is the main process of ${ }^{6} \mathrm{Li}$ formation in the big bang model.

Direct measurements of that process at astrophysical energies are absent due to the smallness of the cross section. The data on the values of the VCs and ANCs for ${ }^{6} \mathrm{Li} \rightarrow \alpha+\mathrm{d}(L=0 ; 2)$ channel obtained by different methods are characterized by a large spread. In the first place it refers to the $D$-state constants.

The procedure of analytic continuation of the two-channel ERE has been applied to $d \alpha$ scattering using several sets of phase shifts. The values of the VCs $G_{L}$ and $\mathrm{ANCs} C_{L}$ for ${ }^{6} \mathrm{Li} \rightarrow \alpha+d(L=0 ; 2)$ have been extracted.

Using the data from the available phase-shifts analysis resulted in $G_{0}^{2}=0.4-0.5 \mathrm{fm}$ and $C_{0}=$ $2.3-2.8 \mathrm{fm}^{-1 / 2}$.

The constants under consideration have also been calculated by solving Faddeev equations in the three-body model of ${ }^{6} \mathrm{Li}(n+p+\alpha)$ giving $G_{0}^{2}=0.3 \mathrm{fm}$ and $C_{0}=2.0 \mathrm{fm}^{-1 / 2}$.

Unfortunately, the poor accuracy of the phase-shift analysis at low energies and the simplified form of the Faddeev equations used did not make it possible to obtain accurate values of the ANC for $L=2: C_{2}=0.02-0.05 \mathrm{fm}^{-1 / 2}$.

Nevertheless, it was established that the sign of $C_{2}$ (relative to $C_{0}$ ) is positive.

One may conclude that the method suggested is operable.

To get the reliable values of VCs and ANCs for ${ }^{6} \mathrm{Li} \rightarrow \alpha+d$ it is desirable:

- to measure more accurately the $d \alpha$ scattering differential cross section at low energies

- to perform the thorough phase-shift analysis of the corresponding data

- to perform Faddeev calculations of ${ }^{6} \mathrm{Li}$ in the 3-body model with realistic pair potentials and account of the Coulomb interaction.

\section{Including inelastic channels}

The procedure described above considers elastic channels only.

On the other hand, low-lying inelastic thresholds might influence the ERE. (The typical example is the deuteron-nucleus scattering). The simplest way to allow for an inelastic channel at $E=E_{0}$ is to include into the ERE an additional term, which is complex at $E>E_{0}$. The form of that term should lead to the correct analytic behavior of scattering amplitudes at the threshold $E=E_{0}$. 
According to the general theory of singularities of Feynman diagrams [12], a singular part of a scattering amplitude near a threshold behaves like $\left(E-E_{0}\right)^{(3 n-5) / 2}$ at $n$ even and like $\left(E-E_{0}\right)^{(3 n-5) / 2} \ln \left(E-E_{0}\right)$ at $n$ odd, where $n=2,3,4, \ldots$ is a number of intermediate particles at the threshold.

Adding to the standard ERE singular terms written above, one obtains for the inelasticity coefficient $\eta_{n}$ for the most important two-particle $(n=2)$ and three-particle $(n=3)$ thresholds:

$$
\begin{gathered}
\eta_{2}=\left\{\frac{K_{0}^{2}+\left(k-d \sqrt{E / E_{0}-1}\right)^{2}}{K_{0}^{2}+\left(k+d \sqrt{E / E_{0}-1}\right)^{2}}\right\}^{1 / 2}, E>E_{0} \\
\eta_{3}=\left\{\frac{\left[K_{0}^{2}+d\left(E / E_{0}-1\right)^{2} \ln \left|E / E_{0}-1\right|\right]^{2}+\left[k-\pi d\left(E / E_{0}-1\right)\right]^{2}}{\left[K_{0}^{2}+d\left(E / E_{0}-1\right)^{2} \ln \left|E / E_{0}-1\right|\right]^{2}+\left[k+\pi d\left(E / E_{0}-1\right)\right]^{2}}\right\}^{1 / 2}, E>E_{0}
\end{gathered}
$$

$K_{0}$ is the standard effective range function and $d>0$ is an additional factor which in the simplest case can be considered as a fitting constant. We plan to apply the above formulas to the $d \alpha$ scattering.

The work has been supported by the Russian Foundation for Basic Research under Grant No. 13-02-00399.

\section{References}

[1] L.D. Blokhintsev, I. Borbeli, E.I. Dolinsky, Part. Nucl. 8, 485 (1977)

[2] A.M. Mukhamedzhanov, R.E. Tribble, Phys. Rev. C 59, 3418 (1999)

[3] L.D. Blokhintsev, Yad. Fiz. 34, 944 (1981)

[4] L.D. Blokhintsev, A.M. Mukhamedzhanov, R. Yarmukhamedov, Eur. Phys. J. A 49, 108 (2013)

[5] M. Viviani, A. Kievsky, M. Rosati, Phys. Rev. C 71, 024006 (2005)

[6] K.M. Nolett, R.B. Wiringa, Phys. Rev. C 83, 041001 (2011)

[7] R.G.Newton, Scattering theory for waves and particles (Springer-Verlag, New York, 1982)

[8] L.D. Blokhintsev, V.I. Kukulin, A.A. Sakharuk, D.A. Savin, E.V. Kuznetsova, Phys. Rev. C 48, 2390 (1993)

[9] Yu.V. Orlov, B.F. Irgaziev, L.I. Nikitina, Phys. At. Nucl. 73, 757 (2010)

[10] J.-M. Sparenberg, P. Capel, D. Baye, Phys. Rev. C 81, 011601 (2010)

[11] L.D. Blokhintsev, Phys. At. Nucl. 74, 1008 (2011)

[12] L.D. Landau, Nucl. Phys. 13, 181 (1959) 